\title{
8 Computing Integral Points on Rational Curves
}

The algorithms that we will present in this chapter are the algorithms of (Alvanos and Poulakis, 2011) with the difference being that the initial algorithms are represented in terms of discrete valuation rings while here we present them in terms of places and in a way that they can be implemented through computational programs. After each algorithm we present a couple of examples and in appendix B we give another example with the code that was used for the implementation step by step.

Throughout the whole chapter $K$ is a number field, $O_{K}$ its ring of integers, $C$ is an affine curve of genus 0 defined by an irreducible polynomial $f(x, y), K(x, y)$ the function field associated to $C, C\left(O_{K}\right)$ the set of integral points of the curve $C, C_{\infty}$ the set of points at infinity and $\Sigma_{\infty}(C)$ the set of places of $K(x, y)$ that are over the infinite place of the rational function field. By $N_{L / K}$ we denote the norm mapping from $L$ to $K$ and in case $K=\mathbb{Q}$ we denote this norm by $N_{L}$.

\subsection{Integral Points on Curves}

By a classical theorem of Siegel, the set of integral points of an algebraic curve $C$ over a number field $K$,

$$
C\left(O_{K}\right)=\left\{(x, y) \in O_{K}^{2} \mid f(x, y)=0\right\},
$$

is finite unless $C$ has genus 0 and $C_{\infty}$ has no more than two elements (see (Lang, 1983), (Siegel, 1969)). Siegel's result does not provide us with any information in the case where $C$ has genus 0 and $\left|C_{\infty}\right| \leq 2$. In this case $C\left(O_{K}\right)$ might have infinitely many points. For $K=\mathbb{Q}$, a necessary and sufficient condition for $C(\mathbb{Z})$ to be infinite can be found in (Poulakis, 2001; Poulakis and Voskos, 2002).

The following theorem completes Siegel's theorem and provides us necessary and sufficient conditions for rational curves to have an infinite number of integral points.

Theorem 8.1.1. Suppose that $C$ has genus 0 . The set $C\left(O_{K}\right)$ is infinite if and only if $C\left(O_{K}\right)$ has a non-singular point and one of the following conditions is satisfied:

(i) $\left|C_{\infty}\right|=1$.

(ii) $\left|C_{\infty}\right|=2$ and the elements of $C_{\infty}$ are defined over $K$ and $K$ is not $\mathbb{Q}$ or an imaginary quadratic field.

(iii) $\left|C_{\infty}\right|=2$ and the elements of $C_{\infty}$ are conjugate over a quadratic extension $K(a)$ of $K$. Furthermore, if $K$ is totally real, then there is a monomorphism

$$
\tau: K(\alpha) \rightarrow \mathbb{C}
$$

such that $\tau(\alpha) \in \mathbb{R}$.

Proof. see (Alvanos et al., 2009). 
For $K=\mathbb{Q}$, we obtain Theorem 5.2 of (Poulakis, 2001).

Corollary 8.1.2. Suppose that $C$ is defined over $\mathbb{Q}$ and has genus 0 . The set $C(\mathbb{Z})$ is infinite if and only if at least one of the following conditions is satisfied:

(i) $\left|C_{\infty}\right|=1$ and $C(\mathbb{Z})$ has a simple point.

(ii) $\left|C_{\infty}\right|=2, C(\mathbb{Z})$ has a simple point and the elements of $C_{\infty}$ are conjugate over a real quadratic field.

In the following sections we present four algorithms for computing each case of the above theorem and the case were $\left|C_{\infty}\right| \geq 3$.

\subsection{Integral Points on Curves with $\left|\Sigma_{\infty}(C)\right| \geq 3$}

In this section we present the algorithm for computing the integral solutions of the equation $f(x, y)=0$ over the number field $K$. The case where $K=\mathbb{Q}$ can be found in (Poulakis and Voskos, 2000). The algorithm that is presented here is efficient and it is based on Hess' algorithm (Hess, 2002) for computing a Riemann-Roch space and in Windanger's algorithm (Wildanger, 2000) for computing unit equations over a field.

\section{Integral Points 3 Algorithm}

Input: An irreducible polynomial $f(x, y)$ over $K$ with at least three discrete valuation rings at infinity.

Output: Integral solutions $(x, y) \in O_{K}^{2}$ of $f(x, y)$.

Step 1: Compute the singular points of $C$.

Step 2: Compute the places at infinity and their degree. If there are at least three places of degree 1 then set $K=L$ go to step 4. Else continue to step 3.

Step 3: Compute a field $L$ by attaching the defining polynomial (or polynomials if one polynomial is not enough) of the residue class field of a place (or places if one place is not enough) of places at infinity of degree greater than 1 and recompute the places at infinity and their degree.

Step 4: Compute the bases $\left\{f_{i}\right\}$ where $f_{i}=a_{i}(x, y) / b_{i}(x) \in L\left(P_{3}-P_{i}\right), i=1,2$ and places $P_{1}, P_{2}, P_{3}$ are of degree 1 .

Step 5: Compute the fields $M_{i} \subset L$ by attaching $f_{i}$ to $K$.

Step 6: Compute $a_{f i}, b_{f i} \in O_{M_{i}}$ such that $a_{f i} f_{i}$ and $b_{f i} / f_{i}$ are integral over $O_{M_{i}}[x]$, where $i=1,2$.

Step 7: Compute $M$ by attaching in $L$ the defining polynomials of $M_{1}$ and $M_{2}$.

Step 8: Compute $c_{1}, c_{2} \in M$ such that $c_{1} f_{1}+c_{2} f_{2}=1$.

Step 9: Compute maximal sets $A_{i}$ of pairwise non associate elements of $O_{M_{i}}$ with norm dividing $N_{M_{i}}\left(a_{f i} b_{f}\right)$.

Step 10: Compute the solutions $\left(u_{1}, u_{2}\right) \in \mathcal{U}$ of the unit equation

$$
\frac{c_{1} k_{1}}{y_{1}} u_{1}+\frac{c_{2} k_{2}}{y_{2}} u_{2}=1
$$


for every $\left(k_{1}, k_{2}\right) \in A_{1} \times A_{2}$.

Step 11: Compute the resultant $\operatorname{Res}_{k_{1}, u_{1}}(f, g)$ of $f(x, y)$ and

$$
g(x, y)=a_{f 1} a_{1}(x, y)-k_{1} u_{1} b_{f 1}(x)
$$

with respect to $y$ for every $\left(k_{1}, k_{2}\right) \in A_{1} \times A_{2}$ and $\left(u_{1}, u_{2}\right) \in \mathcal{U}$.

Step 12: Compute the set $S$ of $s \in O_{K}$ such that $R_{k_{1}, u_{1}}(s)=0$ for some $\left(k_{1}, k_{2}\right) \in A_{1} \times A_{2}$ and $\left(u_{1}, u_{2}\right) \in \mathcal{U}$.

Step 13: Compute all the pairs $(s, t) \in C\left(O_{K}\right)$ where $s \in S$.

Step 14: Output the points from step 13 and step 1.

The proof of correctness can be found in (Alvanos and Poulakis, 2011) along with an example of the algorithm. Next, we give two more examples of the Integral Points 3 algorithm. In the Appendix, we present an example of (Alvanos and Poulakis, 2011) using MAGMA code. All the computations of the following two examples have been carried out by the algebraic systems KASH and MAGMA.

Example In our first example we compute the solutions of $f(x, y)=0$ over $\mathbb{Z}[\sqrt{2}]$ where

$$
f(x, y)=x^{3}+(2-\sqrt{2}) x^{2} y-(3+2 \sqrt{2}) x y^{2}+3 \sqrt{2} y^{3}-x^{2}+3 y^{2}-(\sqrt{2}+1) x y .
$$

The polynomial $f(x, y)$ defines a curve $C$ of genus 0 . It has three points at infinity

$$
p_{1}=(1: 1: 0), p_{2}=(3:-1: 0), p_{3}=(\sqrt{2}: 1: 0)
$$

that correspond to three place at infinity $P_{1}, P_{2}$ and $P_{3}$ of degree 1 . Therefore, we skip step 3 and continue by computing Riemann-Roch spaces $L\left(P_{1}-P_{2}\right)$ and $L\left(P_{1}-P_{3}\right)$. Hence, we get

$$
f_{1}(x, y)=\frac{3 \sqrt{2} y^{2}+(\sqrt{2}-3) x y+3 y-x^{2}+2 x}{2 x}
$$

a function of $L\left(P_{1}-P_{3}\right)$ and

$$
f_{2}(x, y)=\frac{(60 \sqrt{2}+24) y^{2}+((8 \sqrt{2}-52) x+12 \sqrt{2}+60) y-(4 \sqrt{2}+20) x^{2}}{23 x}+1
$$

a function of $L\left(P_{1}-P_{2}\right)$.

Next, define $M_{1}=M_{2}=\mathbb{Q}(\sqrt{2})=M$. If $R_{1}\left(f, g_{1}\right)$ and $R_{2}\left(f, g_{2}\right)$ are the resultants on $y$ over $M$ where

$$
g_{1}(x, y, t)=x f_{1}(x, y)-t x
$$

and

$$
g_{2}(x, y, t)=x f_{2}(x, y)-t x
$$

then

$$
R_{1}\left(f, g_{1}\right)=x^{3} I_{1}(x, t), \quad R_{2}\left(f, g_{2}\right)=x^{3} I_{2}(x, t),
$$


where

$$
\begin{gathered}
I_{1}(x, t)=t^{3}+(-2 \sqrt{2}+2) x t^{2}+1 / 2(-2 \sqrt{2}-5) t^{2}+1 / 4(2 \sqrt{2}-1) t x+ \\
1 / 4(7 \sqrt{2}+5) t+1 / 8(-3 \sqrt{2}-4)
\end{gathered}
$$

and

$$
\begin{gathered}
I_{2}(x, t)=t^{3}+1 / 23(-64 \sqrt{2}+48) t^{2} x+1 / 23(-36 \sqrt{2}-65) t^{2}- \\
1 / 529(704 \sqrt{2}+208) t x+1 / 529(744 \sqrt{2}-213) t+1 / 12167(2124 \sqrt{2}-2835)
\end{gathered}
$$

are irreducible polynomials. Thus, $I_{1}(x, t)$ and $I_{2}(x, t)$ are the polynomials that vanish for $t=f$. Next, we see that the irreducible polynomials of $1 / f_{1}$ and $1 / f_{2}$ over $M(x)$ are

$$
\begin{gathered}
J_{1}(x, t)=t^{3}+((11 \sqrt{2}-16) x+(13 \sqrt{2}-22)) t^{2}+ \\
((-56 \sqrt{2}+80) x+(14 \sqrt{2}-16)) t-12 \sqrt{2}+16
\end{gathered}
$$

and

$$
\begin{gathered}
J_{2}(x, t)=9 t^{3}+((-512 \sqrt{2}-752) x+(348 \sqrt{2}+537)) t^{2}+ \\
((-384 \sqrt{2}-656) x+(-1160 \sqrt{2}-1629)) t+(236 \sqrt{2}+315),
\end{gathered}
$$

respectively. By computation we find that

$$
a_{f 1}=2, \quad b_{f 1}=1, a_{f 2}=23, b_{f 2}=9 .
$$

Hence, we know that the functions $2 f_{1}, 1 / f_{1}, 23 f_{2}$ and $9 / f_{2}$ are integral elements over $O_{M}[x]$.

In step 8 we compute $c_{1}, c_{2} \in K$ such that $c_{1} f_{1}+c_{2} f_{2}=1$. The points

$$
q_{1}=(1,0), \quad q_{2}=\left(\frac{3+\sqrt{2}}{4+4 \sqrt{2}}, \frac{-3-\sqrt{2}}{4+4 \sqrt{2}}\right)
$$

belong to $C$. Thus,

$$
\begin{gathered}
f_{1}\left(q_{1}\right)=\frac{1}{2}, f_{2}\left(q_{1}\right)=\frac{-4 \sqrt{2}+3}{23} \\
f_{1}\left(q_{2}\right)=\frac{1+\sqrt{2}}{4}, f_{2}\left(q_{2}\right)=\frac{5+\sqrt{2}}{23(1+\sqrt{2})} .
\end{gathered}
$$

By solving the linear system

$$
\begin{aligned}
& f_{1}\left(P_{1}\right) c_{1}+f_{2}\left(P_{1}\right) c_{2}=1, \\
& f_{1}\left(P_{2}\right) c_{1}+f_{2}\left(P_{2}\right) c_{2}=1
\end{aligned}
$$

we get that

$$
c_{1}=\frac{24-8 \sqrt{2}}{7}, \quad c_{2}=\frac{-17+8 \sqrt{2}}{7} \text {. }
$$


In step 9 we need to compute two maximal sets $A_{1}$ and $A_{2}$ of pairwise non associate elements of $\mathbb{Z}[\sqrt{2}]$ with norm dividing 4 and $3^{4} 23^{2}$ respectively. Thus, we get

$$
A_{1}=\{\sqrt{2}+1,-\sqrt{2}, 2 \sqrt{2}+2,1,-\sqrt{2}-2,2\}
$$

and

$$
\begin{aligned}
A_{2}= & \{\sqrt{2}+1,3 \sqrt{2}+3,6 \sqrt{2}+7,4 \sqrt{2}+3,9 \sqrt{2}+9,18 \sqrt{2}+21,12 \sqrt{2}+9, \\
& 37 \sqrt{2}+47,23 \sqrt{2}+23,17 \sqrt{2}+7,54 \sqrt{2}+63,36 \sqrt{2}+27, \\
& 111 \sqrt{2}+141,69 \sqrt{2}+69,51 \sqrt{2}+21,333 \sqrt{2}+423,207 \sqrt{2}+207, \\
& 153 \sqrt{2}+63,1,3, \sqrt{2}+5,-\sqrt{2}+5,9,3 \sqrt{2}+15,-3 \sqrt{2}+15, \\
& 10 \sqrt{2}+27,23,-10 \sqrt{2}+27,9 \sqrt{2}+45,-9 \sqrt{2}+45,30 \sqrt{2}+81, \\
& 69,-30 \sqrt{2}+81,90 \sqrt{2}+243,207,-90 \sqrt{2}+243\} .
\end{aligned}
$$

In the next step (step 10), we compute the solutions $\left(u_{1}, u_{2}\right) \in \mathcal{U}$ of the unit equations

$$
\frac{c_{1} k_{1}}{y_{1}} u_{1}+\frac{c_{2} k_{2}}{y_{2}} u_{2}=1
$$

for every $\left(k_{1}, k_{2}\right) \in A_{1} \times A_{2}$. In the table below, the first column contains the couples $\left(k_{1}, k_{2}\right)$ of the unit equation that we have solved and in the second holds their solutions.

\begin{tabular}{c|c}
\hline Couples $\left(k_{1}, k_{2}\right)$ & Solutions $\left(u_{1}, u_{2}\right)$ \\
\hline$(1+\sqrt{2}, 7+6 \sqrt{2})$ & $\begin{array}{c}(-1,-3-2 \sqrt{2}) \\
(-1+\sqrt{2},-3+2 \sqrt{2})\end{array}$ \\
\hline$(1+\sqrt{2}, 63+54 \sqrt{2})$ & $(-7+5 \sqrt{2},-3+2 \sqrt{2})$ \\
\hline$(1+\sqrt{2}, 5+\sqrt{2})$ & $\begin{array}{c}(-1,-7-5 \sqrt{2}) \\
(-1+\sqrt{2}, 1-\sqrt{2})\end{array}$ \\
\hline$(1+\sqrt{2}, 45+9 \sqrt{2})$ & $(-7+5 \sqrt{2}, 1-\sqrt{2})$ \\
\hline$(-\sqrt{2}, 21+18 \sqrt{2})$ & $(-1,3-2 \sqrt{2})$ \\
\hline$(-\sqrt{2}, 47+37 \sqrt{2})$ & $(3+2 \sqrt{2},-1-\sqrt{2})$ \\
\hline$(-\sqrt{2}, 15+3 \sqrt{2})$ & $(-1,-1+\sqrt{2})$ \\
\hline$(-\sqrt{2}, 27+10 \sqrt{2})$ & $(3+2 \sqrt{2},-3-2 \sqrt{2})$ \\
\hline$(2+2 \sqrt{2}, 21+18 \sqrt{2})$ & $(1+\sqrt{2}, 3+2 \sqrt{2})$ \\
\hline$(2+2 \sqrt{2}, 47+37 \sqrt{2})$ & $(3-2 \sqrt{2}, 7-5 \sqrt{2})$ \\
\hline$(2+2 \sqrt{2}, 23+23 \sqrt{2})$ & $(-1+\sqrt{2},-1+\sqrt{2})$ \\
\hline$(2+2 \sqrt{2}, 15+3 \sqrt{2})$ & $(1+\sqrt{2}, 7+5 \sqrt{2})$ \\
\hline$(2+2 \sqrt{2}, 27+10 \sqrt{2})$ & $(3-2 \sqrt{2},-3+2 \sqrt{2})$ \\
\hline$(2+2 \sqrt{2}, 23)$ & $(-1+\sqrt{2}, 1)$ \\
\hline$(1,7+6 \sqrt{2})$ & $(1,-3+2 \sqrt{2})$ \\
\hline$(1,63+54 \sqrt{2})$ & $(-1-\sqrt{2},-3-2 \sqrt{2})$ \\
\hline$(1,5+\sqrt{2})$ & $(-2 \sqrt{2},-3+2 \sqrt{2})$ \\
\hline
\end{tabular}




\begin{tabular}{c|c}
\hline$(1,45+9 \sqrt{2})$ & $(3-2 \sqrt{2}, 1-\sqrt{2})$ \\
\hline$(-2-\sqrt{2}, 21+18 \sqrt{2})$ & $(1-\sqrt{2}, 3-2 \sqrt{2})$ \\
\hline$(-2-\sqrt{2}, 47+37 \sqrt{2}$ & $(1+\sqrt{2},-1-\sqrt{2})$ \\
\hline$(-2-\sqrt{2}, 15+3 \sqrt{2})$ & $(1-\sqrt{2},-1+\sqrt{2})$ \\
\hline$(-2-\sqrt{2}, 27+10 \sqrt{2})$ & $(1+\sqrt{2},-3-2 \sqrt{2})$ \\
\hline$(2,21+18 \sqrt{2})$ & $(3+2 \sqrt{2}, 3+2 \sqrt{2})$ \\
\hline$(2,47+37 \sqrt{2})$ & $(-1+\sqrt{2}, 7-5 \sqrt{2})$ \\
\hline$(2,23+23 \sqrt{2})$ & $(1,-1+\sqrt{2})$ \\
\hline$(2,15+3 \sqrt{2})$ & $(3+2 \sqrt{2}, 7+5 \sqrt{2})$ \\
\hline$(2,27+10 \sqrt{2})$ & $(-1+\sqrt{2},-3+2 \sqrt{2})$ \\
\hline$(2,23)$ & $(1,1)$ \\
\hline
\end{tabular}

For every $\left(k_{1}, k_{2}\right) \in A_{1} \times A_{2}$ and $\left(u_{1}, u_{2}\right) \in \mathcal{U}$, we compute the resultant $\operatorname{Res}_{k_{1}, u_{1}}(f, g)$ on $y$ for $f(x, y)$ and

$$
g(x, y)=3 f_{1}(x, y)-k_{1} u_{1}
$$

(step 11). The resultants are presented in the following table:

\begin{tabular}{c}
\hline Resultants \\
\hline$(90 \sqrt{2}+126) x^{4}+(648 \sqrt{2}+954) x^{3}$ \\
$(36 \sqrt{2}-54) x^{4}+(-36 \sqrt{2}+54) x^{3}$ \\
$(1944 \sqrt{2}-2754) x^{4}+(648 \sqrt{2}-810) x^{3}$ \\
$(162 \sqrt{2}-216) x^{4}$ \\
$(1296 \sqrt{2}+1836) x^{4}+(-4374 \sqrt{2}-6156) x^{3}$ \\
$(1548 \sqrt{2}-2196) x^{4}+(-882 \sqrt{2}+1260) x^{3}$ \\
$(216 \sqrt{2}-252) x^{4}+(-54 \sqrt{2}+108) x^{3}$ \\
$(810 \sqrt{2}+1152) x^{4}+(7776 \sqrt{2}+11016) x^{3}$ \\
\hline
\end{tabular}

Finally, we compute the roots of the above polynomials which are elements of $O_{L}$ are

$$
S=\{0,1,-13 \sqrt{2}+11,8 \sqrt{2}+11\}
$$

Therefore the set

$$
\{(0,0),(1,0),(-13 \sqrt{2}+11, \sqrt{2}-8)\}
$$

are the only solutions of $f(x, y)=0$ over $\mathbb{Z}[\sqrt{2}]$.

\section{Example Let}

$$
f(x, y)=(y-2 x)^{2}(y+\sqrt[3]{-2} x)+(\sqrt[3]{-2})^{2} y
$$

The curve $C$ that is defined by $f(x, y)$ over $K=\mathbb{Q}(\sqrt[3]{-2})$ is of genus 0 and has a singular point $p=(1 / 2: 1: 0)$ that is at infinity. Besides $p, C$ has another point at infinity given by $p_{1}=(1:-\sqrt[3]{-2}: 0)$. Using MAGMA we find that over $p_{1}$ there is a unique place $P_{1}$ of degree 1 , while over the singular point $p$ there is a place of degree 2 .

According to step 3, in order to define two places $P_{2}$ and $P_{3}$ of degree 1 over the singular point we define the field $L=\mathbb{Q}(\sqrt[3]{-2})(\theta)$ where

$$
\theta^{2}+\left(-2(\sqrt[3]{-2})^{2}+2 \sqrt[3]{-2}+6\right) \theta-(\sqrt[3]{-2})^{2}-2 \sqrt[3]{-2}+21=0
$$


Moreover, we know that the field $L$ is the field $\mathbb{Q}(\eta)$ where $\eta$ is a root of the polynomial

$$
z^{6}+18 z^{5}+147 z^{4}+792 z^{3}+3171 z^{2}+8190 z+9525 .
$$

Using MAGMA again, we find that

$$
f_{1}=-A(\eta) y+2 A(\eta) x+1
$$

is a function of $L\left(P_{1}-P_{3}\right)$ and

$$
f_{2}=A(\eta) y-2 A(\eta) x+1
$$

is a function of $L\left(P_{1}-P_{2}\right)$ where

$$
A(\eta)=\frac{1}{26660}\left(146 \eta^{5}+2048 \eta^{4}+12687 \eta^{3}+59297 \eta^{2}+195721 \eta+276975\right) .
$$

Next, according to the Denominators algorithm we compute the resultants $R_{1}\left(g_{1}, f\right)$ and $R_{2}\left(g_{2}, f\right)$ on $y$ of the polynomials

$$
g_{1}(x, y, t)=f_{1}(x, y)-t, f(x, y)
$$

and

$$
g_{2}(x, y, t)=f_{2}(x, y)-t, f(x, y),
$$

respectively. Thus, we get

$$
\begin{aligned}
& R_{1}\left(g_{1}, f\right)=t^{3}+c_{1} t^{2} x-3 t^{2}-2 c_{1} t x+c_{2} t+c_{3}, \\
& R_{2}\left(g_{2}, f\right)=t^{3}-c_{1} t^{2} x-3 t^{2}+2 c_{1} t x+c_{2} t+c_{3},
\end{aligned}
$$

where

$$
\begin{aligned}
& c_{1}=\frac{1}{133300}\left(263 \eta^{5}+3324 \eta^{4}+27556 \eta^{3}+128226 \eta^{2}+481803 \eta+1114260\right), \\
& c_{2}=\frac{1}{133300}\left(428 \eta^{5}+4269 \eta^{4}+22036 \eta^{3}+96406 \eta^{2}+250368 \eta+398085\right), \\
& c_{3}=-\frac{1}{133300}\left(428 \eta^{5}+4269 \eta^{4}+22036 \eta^{3}+96406 \eta^{2}+250368 \eta+131485\right) .
\end{aligned}
$$

Thus, $R_{1}\left(g_{1}, f\right)$ and $R_{2}\left(g_{2}, f\right)$ are the polynomials that vanish for $t=f$.

Next, according to step 6, we get

$$
a_{f 1}=2, \quad b_{f 1}=1, a_{f 2}=2, b_{f 2}=1 .
$$

Hence, we see that the functions $2 f_{1}, 1 / f_{1}, 2 f_{2}$ and $1 / f_{2}$ are integral elements over $O_{L}[x]$.

Now, let $q_{1}=(0,0)$ and $q_{2}=(0, \sqrt{-1} \sqrt[3]{-2})$ be two points of $C$. We have that

$$
f_{1}\left(q_{1}\right)=f_{2}\left(q_{1}\right)=1 \text {, }
$$


and

$$
\begin{gathered}
f_{1}\left(q_{2}\right)=-A(\eta) \sqrt{-1} \sqrt[3]{-2}+1 \\
f_{2}\left(q_{2}\right)=A(\eta) \sqrt{-1} \sqrt[3]{-2}+1
\end{gathered}
$$

The solutions of

$$
\begin{aligned}
& c_{1} f_{1}\left(q_{1}\right)+c_{2} f_{2}\left(q_{1}\right)=1, \\
& c_{1} f_{1}\left(q_{2}\right)+c_{2} f_{2}\left(q_{2}\right)=1
\end{aligned}
$$

gives us $c_{1}=c_{2}$ and $c_{1}+c_{2}=1$ and therefore

$$
c_{1}=c_{2}=\frac{1}{2} .
$$

The two maximal sets $A_{1}$ and $A_{2}$ of pairwise non associate elements of $O_{L}$ are the same. Thus, the elements of the maximal set $A$ with norm dividing $2^{6}$ are

$$
\begin{aligned}
& 1, \frac{1}{26660}\left(-168 \eta^{5}-2174 \eta^{4}-11951 \eta^{3}-50611 \eta^{2}-178193 \eta-288125\right), \\
& \frac{1}{26660}\left(-156 \eta^{5}-3923 \eta^{4}-42042 \eta^{3}-234092 \eta^{2}-665816 \eta-763135\right), \\
& \frac{1}{13330}\left(663 \eta^{5}+18339 \eta^{4}+195341 \eta^{3}+1021551 \eta^{2}+2656428 \eta+2778440\right) .
\end{aligned}
$$

By Trager's algorithm we obtain an integral basis of $L$ that consists of the elements

$$
\begin{gathered}
\omega_{0}=1, \omega_{1}=\eta, \omega_{2}=\eta^{2}, \\
\omega_{3}=\frac{1}{4}\left(\eta^{3}+\eta^{2}+3 \eta+1\right), \\
\omega_{4}=\frac{1}{4}\left(\eta^{4}+2 \eta^{2}+2 \eta+3\right), \\
\omega_{5}=\frac{1}{266600}\left(\eta^{5}+58173 \eta^{4}+30262 \eta^{3}+260752 \eta^{2}+27681 \eta+132595\right) .
\end{gathered}
$$

So we can represent an integral element $z$ as follows:

$$
z=\left[z_{0}, z_{1}, z_{2}, z_{3}, z_{4}, z_{5}\right]
$$

where $z_{i}$ are the coefficients of $\omega_{i}$.

Next, there is a unique element $\left(k_{1}, k_{2}\right) \in A \times A$ for which the unit equation

$$
k_{1} u_{1}+k_{2} u_{2}=4
$$

has solutions and this is

$$
\left(\frac{k}{13330}, \frac{k}{13330}\right)
$$

where

$$
k=663 \eta^{5}+18339 \eta^{4}+195341 \eta^{3}+1021551 \eta^{2}+2656428 \eta+2778440 .
$$

The solutions $\left(u_{1}, u_{2}\right)$ of the above equation are as follows: 


$$
\begin{gathered}
\hline u_{1}=[-86753,-211379,133351,141374,272463,-312238] \\
u_{2}=[-6627,-16411,10387,11012,21207,-24302] \\
\hline u_{1}=[-83562,-203834,128624,136363,262790,-301152] \\
\left.u_{2}=[-9818,-23956,15114,16023,30880,-35388]\right) \\
\hline u_{1}=[-46405,-114534,72494,76854,148006,-169604] \\
v[-46975,-113256,71244,75532,145664,-166936] \\
\hline u_{1}=[-46690,-113895,71869,76193,146835,-168270] \\
u_{2}=[-46690,-113895,71869,76193,146835,-168270] \\
\hline u_{1}=[-46975,-113256,71244,75532,145664,-166936] \\
u_{2}=[-46405,-114534,72494,76854,148006,-169604] \\
\hline u_{1}=[-9818,-23956,15114,16023,30880,-35388] \\
\frac{u_{2}=[-83562,-203834,128624,136363,262790,-301152]}{u_{1}=[-6627,-16411,10387,11012,21207,-24302]} \\
\frac{u_{2}=[-86753,-211379,133351,141374,272463,-312238]}{}
\end{gathered}
$$

For all $u_{1}$ of the above table we solve the system

$$
\begin{aligned}
f(x, y) & =0 \\
2(A(\eta)(-y+2 x)+1) & =k_{1} u_{1}
\end{aligned}
$$

By solving the above system we get the solutions $(x, y)$ of $f(x, y)=0$ over $\mathbb{Z}[\sqrt[3]{-2}]$. Those are

$$
\begin{gathered}
(0,0),\left(-2(\sqrt[3]{-2})^{2}+3 \sqrt[3]{-2}-3,-2(\sqrt[3]{-2})^{2}+4 \sqrt[3]{-2}-4\right) \\
\left(2(\sqrt[3]{-2})^{2}-3 \sqrt[3]{-2}+3,2(\sqrt[3]{-2})^{2}-4 \sqrt[3]{-2}+4\right)
\end{gathered}
$$

Obviously this set of the solutions is finite (Siegel's theorem (Siegel, 1969), (Lang, 1978)).

\subsection{Integral Points on Curves with $\left|\Sigma_{\infty}(C)\right|=2$ (i)}

As was mentioned, the case where $\Sigma_{\infty}(C)$ has two valuations is divided into two cases. In this section we present the case where the valuations are defined over $K$.

\section{Integral Points 2i Algorithm}

Input: An irreducible polynomial $f(x, y)$ of degree $n$ over $K$ with two discrete valuation rings at infinity.

Output: Integral solutions $(x, y) \in O_{K}^{2}$ of $f(x, y)$.

Step 1: Compute the singular points of $C$.

Step 2: Compute the parametrization of $f$

$$
\begin{aligned}
\phi: \mathbb{A} & \rightarrow C \\
t & \longmapsto\left(\frac{u(t)}{w(t)}, \frac{v(t)}{w(t)}\right)
\end{aligned}
$$

where $u(t)$ and $v(t)$ are polynomials over $O_{K}$. If such polynomials do not exist, then go to Step 12 . 
Step 3: Change coordinates such that

$$
\begin{aligned}
\psi: \mathbb{A} & \rightarrow C \\
t & \longmapsto\left(\frac{p(t)}{a t^{m}}, \frac{q(t)}{a t^{m}}\right)
\end{aligned}
$$

Step 4: For

$$
\begin{gathered}
p(t)=a_{0} t^{n}+a_{1} t^{n-1}+\cdots+a_{n}, \\
q(t)=b_{0} t^{n}+b_{1} t^{n-1}+\cdots+b_{n}
\end{gathered}
$$

compute $a_{0}^{\prime}, b_{0}^{\prime}$ such that $a_{0}=a_{0}^{\prime} A_{0}, b_{0}=b_{0}^{\prime} B_{0}$, where $a_{0}^{\prime}, b_{0}^{\prime} \in \mathbb{Z}$ and $A_{0}, B_{0}$ are equal to 1 or they are not rational integers.

Step 5: Set $L_{A}=\mathbb{Q}\left(A_{0}\right)$ and $L_{B}=\mathbb{Q}\left(B_{0}\right)$.

Step 6: Compute

$$
\begin{gathered}
d_{0}=\operatorname{gcd}\left(a_{0}^{\prime} N_{L_{A}}\left(A_{0}\right), b_{0}^{\prime} N_{L_{B}}\left(B_{0}\right)\right), \\
d_{n}=\operatorname{gcd}\left(a_{n}^{\prime} N_{K}\left(A_{n}\right), b_{n}^{\prime} N_{K}\left(B_{n}\right)\right) .
\end{gathered}
$$

Step 7: Compute the resultant $R=\operatorname{Res}(\tilde{p}, \tilde{q})$ of the polynomials

$$
\begin{aligned}
& \tilde{p}(t)=a_{0} t^{n}+a_{1} d_{0} t^{n-1}+\cdots+a_{n} d_{0}^{n}, \\
& \tilde{q}(t)=b_{0} t^{n}+b_{1} d_{0} t^{n-1}+\cdots+b_{n} d_{0}^{n} .
\end{aligned}
$$

If $N_{K}\left(a t^{m}\right)$ does not divides $N_{K}(R)$ go to step 13.

Step 8: Compute maximal set $S$ of pairwise non associate elements $s \in O_{K}$ with norm dividing $\operatorname{gcd}\left(d_{0}^{N \operatorname{deg} K} d_{n}, N_{K}(R)\right)$.

Step 9: Compute a basis $\left\{e_{1}, \ldots, e_{r}\right\}$ of the unit group $U_{K}$.

Step 10: Compute the order $\sigma(i, s)$ of the image of the class of $e_{i}$ in the unit group $O_{K} / I$ where $I$ is the principal ideal $\left(a d_{0}^{m} s^{n-m}\right)$ for every $s \in S$.

Step 11: Compute the set $A$ of $r+1$-tuples $\left(s, l_{1}, \ldots, l_{r}\right), \forall s \in S$, with $0 \leq l_{i}<\sigma(i, s)$ $(i=1, \ldots, r)$ such that

$$
\begin{aligned}
& x\left(s, l_{1}, \ldots, l_{r}\right)=\frac{\tilde{p}\left(s e_{1}^{l_{1}} \cdots e_{r}^{l_{r}}\right)}{d_{0}^{m} a\left(s e_{1}^{l_{1}} \cdots e_{r}^{l_{r}}\right)^{n-m}}, \\
& y\left(s, l_{1}, \ldots, l_{r}\right)=\frac{\tilde{q}\left(s e_{1}^{l_{1}} \cdots e_{r}^{l_{r}}\right)}{d_{0}^{m} a\left(s e_{1}^{l_{1}} \cdots e_{r}^{l_{r}}\right)^{n-m}},
\end{aligned}
$$

are elements $O_{K}$.

Step 12: Compute $(x, y)$, where

$$
\begin{aligned}
& x=x\left(s, l_{1}+z_{1} \sigma(1, s), \ldots, l_{r}+z_{r} \sigma(r, s)\right), \\
& y=y\left(s, l_{1}+z_{1} \sigma(1, s), \ldots, l_{r}+z_{r} \sigma(r, s)\right),
\end{aligned}
$$

$\left(s, l_{1}, \ldots, l_{r}\right) \in A$ and $z_{1}, \ldots, z_{r} \in \mathbb{Z}$.

Step 13: Output the points from step 12 and step 1. 
The proof of correctness can be found in (Alvanos and Poulakis, 2011). Next we will see an example with no solutions. In the appendix we present code for an example with solutions.

Example Let $C$ be the curve defined by

$$
f(x, y)=100 x^{3}+180 \sqrt{-5} x^{2}-75 x y^{2}-540 x+25 y^{3}-45 \sqrt{-5} y^{2}+27 \sqrt{-5}
$$

over $K=\mathbb{Q}(\sqrt{-5})$. The ring of integers of $K$ is $\mathbb{Z}[\sqrt{-5}]$. The only singular point of $C$ is at infinity and it is $(1 / 2: 1: 0)$. Using MAPLE we obtain a parametrization for $C$ given by the birational morphism

$$
\begin{aligned}
\phi: \mathbb{A} & \longrightarrow C, \\
t & \longmapsto\left(\frac{u(t)}{w(t)}, \frac{v(t)}{w(t)}\right),
\end{aligned}
$$

where

$$
\begin{aligned}
u(t)= & -81569(906 \sqrt{-5}-1895)[31409+(1980 \sqrt{-5}+12885) t \\
& \left.-(2790 \sqrt{-5}+39540) t^{2}+(-1000+765 \sqrt{-5}) t^{3}\right], \\
v(t)= & -31409(3985+906 \sqrt{-5})[163138+(-95640+21744 \sqrt{-5}) t \\
& \left.+(5430-14130 \sqrt{-5}) t^{2}+(1035 \sqrt{-5}+2200) t^{3}\right], \\
w(t)= & 38430010815\left(392+(84 \sqrt{-5}-140) t-(15 \sqrt{-5}+10) t^{2}\right) .
\end{aligned}
$$

We change coordinates by setting $w=(6 \sqrt{-5}+10) t-5$. Thus, we obtain the following birational morphism

$$
\begin{aligned}
\psi: \mathbb{A} & \longrightarrow C, \\
w & \longmapsto\left(\frac{p(w)}{r(w)}, \frac{q(w)}{r(w)}\right),
\end{aligned}
$$

where

$$
\begin{aligned}
p(w)= & 675 \sqrt{-5}+9 \sqrt{-5} w+w^{3} \\
q(w)= & (8946 \sqrt{-5}-56320)+(5760 \sqrt{-5}-3840) w \\
& +(360 \sqrt{-5}+600) w^{2}+25 w^{3} \\
r(w)= & 15 w^{2} .
\end{aligned}
$$

By computation, $d_{0}=d_{n}=1$ and therefore $\tilde{p}(w)=p(w)$ and $\tilde{q}(w)=q(w)$. The norm of the resultant of $p(w), q(w)$ is equal to

$$
5^{3} \times 547 \times 184649 \times 103297178694980827
$$

which is not divisible by 15 . Thus, our algorithm implies that the equation $f(x, y)=0$ has no solution in $\mathbb{Z}[\sqrt{-5}]$, since it doesn't pass our check at step 8 and the singular point of $C$ is at infinity. 


\subsection{Integral Points on Curves with $\left|\Sigma_{\infty}(C)\right|=2$ (ii)}

Next, we present an algorithm for the case where the number of elements of $\Sigma_{\infty}(C)$ is two and they are not defined over $K$.

\section{Integral Points 2ii Algorithm}

Input: An irreducible polynomial $f(x, y)$ of degree $n$ over $K$ with two discrete valuation rings at infinity defined over a quadratic extension of $K$.

Output: Integral solutions $(x, y) \in O_{K}^{2}$ of $f(x, y)$.

Step 1: Compute the singular points of $C$.

Step 2: Compute the parametrization of $f$

$$
\begin{aligned}
\phi: \mathbb{A} & \rightarrow C \\
t & \longmapsto\left(\frac{u(t)}{w(t)}, \frac{v(t)}{w(t)}\right)
\end{aligned}
$$

where $u(t), v(t)$ and $w(t)$ are polynomials over $O_{K}$. If there are no such polynomials, then go to Step 14 .

Step 3: Change coordinates such that

$$
\begin{aligned}
\psi: \mathbb{A} & \rightarrow C \\
t & \longmapsto\left(\frac{p(t)}{a\left(t^{2}-\delta\right)^{m / 2}}, \frac{q(t)}{a\left(t^{2}-\delta\right)^{m / 2}}\right)
\end{aligned}
$$

Step 4: Set $L=K(\sqrt{\delta})$ and change coordinates so that

$$
\begin{aligned}
\omega: \mathbb{A} & \rightarrow C \\
t & \longmapsto\left(\frac{p(t)}{b t^{m / 2}}, \frac{q(t)}{b t^{m / 2}}\right)
\end{aligned}
$$

where $b \in O_{L}$

Step 5: For

$$
\begin{aligned}
& p(t)=a_{0} t^{n}+a_{1} t^{n-1}+\cdots+a_{n}, \\
& q(t)=b_{0} t^{n}+b_{1} t^{n-1}+\cdots+b_{n}
\end{aligned}
$$

compute $a_{0}^{\prime}, b_{0}^{\prime}$ such that $a_{0}=a_{0}^{\prime} A_{0}, b_{0}=b_{0}^{\prime} B_{0}$, where $a_{0}^{\prime}, b_{0}^{\prime} \in \mathbb{Z}$ and $A_{0}, B_{0}$ are equal to 1 or they are not rational integers.

Step 6: Set $L_{1}=\mathbb{Q}(A)$ and $L_{2}=\mathbb{Q}(B)$.

Step 7: Compute $d=\operatorname{gcd}\left(a_{0}^{\prime} N_{L_{1}}(A), b_{0}^{\prime} N_{L_{2}}(B)\right)$.

Step 8: Compute the resultant $R=\operatorname{Res}(\tilde{p}, \tilde{q})$ of polynomials

$$
\begin{aligned}
& \tilde{p}(t)=a_{0} T^{n}+a_{1} d t^{n-1}+\cdots+a_{n} d^{n}, \\
& \tilde{q}(t)=b_{0} T^{n}+b_{1} d t^{n-1}+\cdots+b_{n} d^{n} .
\end{aligned}
$$

If $N_{L}\left(b d^{n / 2}\right)$ does not divide $N_{L}(R)$ go to step 14 .

Step 9: Compute maximal set $S$ of pairwise non associate elements of $O_{L}$ with norm equal to $d^{2}$. 
Step 10: Compute a basis $\left\{e_{1}, \ldots, e_{r}\right\}$ of the unit group with elements $N_{L / K}(\zeta)=1$, where $\zeta \in U_{L}$.

Step 11: Compute the order $\sigma(i, s)$ of the image of the class of $e_{i}$ in the unit group $O_{K} / I$ where $I$ is the principal ideal $\left(b(d s)^{n / 2}\right)$ for every $s \in S$.

Step 12: Compute the set $A$ of $r+1$-tuples $\left(s, l_{1}, \ldots, l_{r}\right), \forall s \in S$, with $0 \leq l_{i}<\sigma(i, s)$ $(i=1, \ldots, r)$ such that

$$
\begin{aligned}
& x\left(s, l_{1}, \ldots, l_{r}\right)=\frac{\tilde{p}\left(s e_{1}^{l_{1}} \cdots e_{r}^{l_{r}}\right)}{b\left(d s e_{1}^{l_{1}} \cdots e_{r}^{l_{r}}\right)^{n / 2}}, \\
& y\left(s, l_{1}, \ldots, l_{r}\right)=\frac{\tilde{q}\left(s e_{1}^{l_{1}} \cdots e_{r}^{l_{r}}\right)}{b\left(d s e_{1}^{l_{1}} \cdots e_{r}^{l_{r}}\right)^{n / 2}},
\end{aligned}
$$

are elements $O_{K}$.

Step 13: Compute $(x, y)$, where

$$
\begin{aligned}
& x=x\left(s, l_{1}+z_{1} \sigma(1, s), \ldots, l_{r}+z_{r} \sigma(r, s)\right), \\
& y=y\left(s, l_{1}+z_{1} \sigma(1, s), \ldots, l_{r}+z_{r} \sigma(r, s)\right),
\end{aligned}
$$

$\left(s, l_{1}, \ldots, l_{r}\right) \in A$ and $z_{1}, \ldots, z_{r} \in \mathbb{Z}$.

Step 14: Output the points from step 13 and step 1.

Next we give two examples for computing integral solutions using the Integral Points 2ii algorithm and a third example exists can be found in the appendix. The first one is a Pell equation over $\mathbb{Z}[\sqrt{3}]$.

Example In this example we will compute the solutions of the equation

$$
x^{2}-2 y^{2}=1
$$

in $\mathbb{Z}[\sqrt{3}]$. The curve $C$ defined by

$$
f(x, y)=x^{2}-2 y^{2}-1
$$

is of genus 0 and has no singular points.

A parametrization of the curve $C$ is given by the morphism

$$
\begin{aligned}
\phi: \mathbb{A} & \rightarrow C \\
t & \longmapsto\left(\frac{t^{2}+2}{t^{2}-2}, \frac{2 t}{t^{2}-2}\right)
\end{aligned}
$$

where it is obvious we do not need to execute step 3. Obviously $\delta=2$ and therefore we set $L=K(\sqrt{2})$. By changing the coordinates we see that

$$
\begin{aligned}
\psi: \mathbb{A} & \rightarrow C \\
t & \longmapsto\left(\frac{\sqrt{2}\left(t^{2}+1\right)}{2 \sqrt{2} t}, \frac{t^{2}-1}{2 \sqrt{2} t}\right) .
\end{aligned}
$$


We have

$$
d=\operatorname{gcd}\left(1, N_{\mathbb{Q}(\sqrt{2})}(\sqrt{2})\right)=1
$$

The resultant $R$ of the polynomials

$$
p(t)=\sqrt{2} t^{2}+\sqrt{2}, q(t)=t^{2}-1
$$

is 8. Since $N_{L}(2 \sqrt{2}) \mid R^{4}$, we continue to the following steps of our algorithm.

A basis of the unit subgroup of $U_{L}$ consisting of $\zeta \in U_{L}$ with $N_{L / K}(\zeta)=1$ is given by the set

$$
\{-1,-2 \sqrt{2}+3, \sqrt{3}-\sqrt{2}\} \text {. }
$$

The orders of the images of $3-2 \sqrt{2}$ and $\sqrt{3}-\sqrt{2}$ in the unit group of $\left(O_{L} /(2 \sqrt{2})\right)$ are 2.

Finally, we compute the quantities

$$
\frac{t^{2}+1}{2 t}, \frac{t^{2}-1}{2 \sqrt{2} t}
$$

where

$$
t= \pm(3-2 \sqrt{2})^{k}(\sqrt{3}-\sqrt{2})^{l}
$$

with $k, l \in\{0,1\}$, and we see that they are elements of $\mathbb{Z}[\sqrt{3}]$.

Thus, the solutions of our Pell equation are the couples $(x, y)$, where

$$
\begin{aligned}
& x= \pm \frac{(3-2 \sqrt{2})^{k}(\sqrt{3}-\sqrt{2})^{l}+(3+2 \sqrt{2})^{k}(\sqrt{3}+\sqrt{2})^{l}}{2}, \\
& y= \pm \frac{(3-2 \sqrt{2})^{k}(\sqrt{3}-\sqrt{2})^{l}-(3+2 \sqrt{2})^{k}(\sqrt{3}+\sqrt{2})^{l}}{2 \sqrt{2}},
\end{aligned}
$$

and $k, l \in \mathbb{Z}$.

The following is an example of an equation with no integral solutions.

Example Let $a=(1+\sqrt{-3}) / 2$ and

$$
\begin{aligned}
f(x, y)= & 5625 x^{4}+1500000 a x^{2} y^{2}-(100000000 a-100000000) y^{4} \\
& -3500 x^{3}+660000 a x y^{2}+750 x^{2}-19200 a y^{2}-60 x+1
\end{aligned}
$$

We will show that the equation $f(x, y)=0$ has no solution in $\mathbb{Z}[a]$.

Let $C$ be the curve defined by $f(x, y)$. The curve is rational and has no singular points. A parametrization of $C$ is given by the morphism

$$
\begin{aligned}
\phi: \mathbb{A} & \rightarrow C \\
t & \longmapsto\left(\frac{16 t^{4}+1}{80 t^{4}-120 t^{2}+45}, \frac{2 a t}{80 t^{4}-120 t^{2}+45}\right)
\end{aligned}
$$

Setting $t=2 t$, we obtain the parametrization 


$$
\begin{aligned}
\psi: \mathbb{A} & \rightarrow C \\
t & \longmapsto\left(\frac{t^{4}+1}{5\left(t^{2}-3\right)^{2}}, \frac{a t}{5\left(t^{2}-3\right)^{2}}\right)
\end{aligned}
$$

So $\delta=3$ and $L=K(\sqrt{3})$. Finally, we get the parametrization

$$
\begin{aligned}
\omega: \mathbb{A} & \rightarrow C \\
t & \longmapsto\left(\frac{p(t)}{720 t^{2}}, \frac{q(t)}{720 t^{2}}\right)
\end{aligned}
$$

where

$$
\begin{aligned}
& p(t)=2\left(5 t^{4}+16 t^{3}+30 t^{2}+16 t+5\right), \\
& q(t)=a \sqrt{3}\left(t^{4}-2 t^{3}+2 t-1\right) .
\end{aligned}
$$

Then $d=\operatorname{gcd}\left(10, N_{L}(\sqrt{3})\right)=1$.

Next, we compute the resultant $R$ of the polynomials $p(T, 1)$ and $q(T, 1)$. We have $R=-429981696 a$. Since $N_{K}(R)=2^{32} 3^{16}$ and $N_{K}(720)=2^{8} 3^{4} 5^{2}$, we deduce that $N_{K}(720)$ does not divide $N_{K}(R)$ and so the equation $f(x, y)=0$ does not have any solution in $\mathbb{Z}[a]$.

\subsection{Integral Points on Curves with $\left|\Sigma_{\infty}(C)\right|=1$}

In this final section, we will consider the case where the curve $C$ has $\left|\Sigma_{\infty}(C)\right|=1$. The following algorithm computes the integral solutions of $f(x, y)=0$ over $K$ where $f(x, y)$ is the defining polynomial of $C$.

\section{Integral Points 1 Algorithm}

Input: An irreducible polynomial $f(x, y)$ of degree $n$ over $K$ with one discrete valuation ring at infinity.

Output: Integral solutions $(x, y) \in O_{K}^{2}$ of $f(x, y)$

Step 1: Compute the singular points of $C$.

Step 2: Compute the parametrization of $f$

$$
\begin{aligned}
\phi: \mathbb{A} & \rightarrow C \\
t & \longmapsto\left(\frac{u(t)}{w(t)}, \frac{v(t)}{w(t)}\right)
\end{aligned}
$$

If there are no such polynomials, then go to Step 8 .

Step 3: Change coordinates such that

$$
\begin{aligned}
\psi: \mathbb{A} & \rightarrow C \\
t & \longmapsto\left(\frac{p(t)}{d}, \frac{q(t)}{d}\right)
\end{aligned}
$$


Step 4: Compute $\delta$ the gcd of the norms of the coefficients of the leading polynomials of $p(t)$ and $q(t)$.

Step 5: Compute

$$
\tilde{p}(t)=(\delta D)^{\operatorname{deg} p} p(t / \delta D)
$$

and

$$
\tilde{q}(t)=(\delta D)^{\operatorname{deg} q} q(t / \delta D),
$$

where $D=1$ if $d \in \mathbb{Z}$ and $D=N_{K}(d) / d$, otherwise.

Step 6: Set $\tilde{d}=d$, if $d \in \mathbb{Z}$ and $\tilde{d}=N_{K}(d)$, otherwise.

Step 7: Compute the elements $s$ of the set $S \subset O_{K}$ where

$$
\tilde{p}(s) \equiv 0 \bmod \tilde{d} \delta^{\operatorname{deg} \tilde{p}}, \tilde{q}(s) \equiv 0 \bmod \tilde{d} \delta^{\operatorname{deg} \tilde{q}} .
$$

Step 8: Compute the couples

$$
(x, y)=\left(\frac{\tilde{p}(s)}{\tilde{d} \delta^{\operatorname{deg} \tilde{p}}}, \frac{\tilde{q}(s)}{\tilde{d} \delta^{\operatorname{deg} \tilde{q}}}\right)
$$

for all $s \in S$.

Step 9: Output couples $(x, y)$ from steps 8 and 1 .

As in all previous cases the proof of correctness and an example can be found in (Alvanos and Poulakis, 2011). Next we give an example without solutions while in the appendix we present code for the example of (Alvanos and Poulakis, 2011) with solutions. The computations have been carried out by MAGMA http://magma.maths.usyd.edu.au/magma/ and MAPLE http://www.maplesoft.com/.

Example Let $a$ be an algebraic integer such that $a^{3}-a-2=0$ and

$$
f(x, y)=f_{3}(x, y)+f_{2}(x, y)+f_{1}(x, y)+f_{0}(x, y),
$$

where

$$
\begin{aligned}
& f_{3}(x, y)=216 x^{3}-324 a x^{2} y+162 a^{2} x y^{2}-(27 a+54) y^{3}, \\
& f_{2}(x, y)=-(108 a+216) x^{2}+\left(108 a^{2}+216 a\right) x y-\left(54 a^{2}+27 a+54\right) y^{2}, \\
& f_{1}(x, y)=\left(18 a^{2}+72 a+72\right) x-\left(36 a^{2}+45 a+342\right) y, \\
& f_{0}(x, y)=-114 a^{2}-13 a-10 .
\end{aligned}
$$

We will show that the equation $f(x, y)=0$ has no solution in integers of $K$. Set $K=\mathbb{Q}(a)$ and denote by $C$ the curve defined by $f(x, y)$. The ring of integers of $K$ is $O_{K}=\mathbb{Z}[a]$. The point at infinity $(a / 2: 1: 0)$ is its only singular point. A parametrization of $C$ is given by

$$
\begin{aligned}
\phi: \mathbb{A} & \rightarrow C \\
t & \longmapsto\left(\frac{a t^{3}+3 t}{3}, \frac{2 t^{3}-a^{2}}{3}\right) .
\end{aligned}
$$


We have that

$$
\delta=\operatorname{gcd}\left(N_{K}(a), N_{K}(2)\right)=2
$$

and since $d=3$ we set $D=1$. Thus, we get

$$
\begin{aligned}
& p(t)=a t^{3}+12 t \\
& q(t)=2 t^{3}-8 a^{2}
\end{aligned}
$$

Next (step 7) we have to find the elements $s \in O_{K}$ satisfying

$$
\begin{aligned}
a s^{3}+12 s & \equiv \bmod 24 \\
2 t^{3}-8 a^{2} & \equiv \bmod 24
\end{aligned}
$$

MAPLE return an empty set $S$ and since the unique singular point is at infinity we deduce that $f(x, y)=0$ has no integral solutions. 